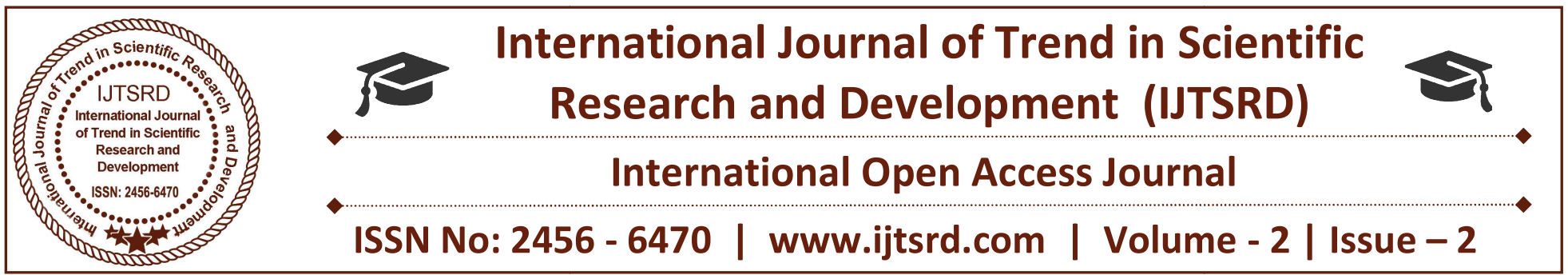

\title{
Micropropagation of Atropa acuminata Royle from in vitro petiole explant
}

\author{
Farhana Maqbool, Seema Singh, Mahroofa Jan
}

Plant Tissue Culture Laboratory, Department of Botany, University of Kashmir, Hazratbal, Srinagar, J\&K, India

\section{ABSTRACT}

During the present study, in vitro petiole explants of Atropa acuminata were subjected to in vitro studies so as to develop efficient micropropagation protocols for its regeneration. In vitro petiole explant produced maximum amount of callus on MS medium supplemented with BAP $(3 \mathrm{mg} / \mathrm{l})$ in $80 \%$ cultures within 20 days. Shoot regeneration was obtained after sub-culturing the callus on MS medium supplemented with BAP with mean shoot number of $8.6 \pm 4.22 \mathrm{~cm}$ and a mean shoot length of $2.3 \pm 0.20 \mathrm{~cm}$ in $100 \%$ cultures within 13 days. Root regeneration was obtained on MS medium augmented with IBA $(0.5 \mathrm{mg} / \mathrm{l})$ with a mean number of roots $6.0 \pm 2.50 \mathrm{~cm}$ and mean root length of $1.3 \pm 0.11 \mathrm{~cm}$ with $60 \%$ response within 32 days.

Keywords: Atropa acuminata, micropropagation, explant, callus, shoot regeneration, root regeneration

\section{Introduction}

Atropa acuminata is commonly known as Indian Belladonna. It is a perennial plant and grows about $1.6 \mathrm{~m}$ tall. It has simple leaves which are ovate with entire margins. The flowers are solitary, bell-shaped and yellowish white in colour. They are hermaphrodites and are pollinated by insects (Nasir, 1972). Flowering period is from June to July and the seeds ripe from August to October. The black fruits are berries. The rhizome of this plant has been traditionally used as a sedative (Rhodes et al., 1978) antidote in cases of mushroom or toadstool poisoning, analgesic, antispasmodic, hallucinogenic, mydriatic, narcotic (Grieve, 1984) diuretic, anodyne (Chiej, 1984), arthritis related inflammatory disorders, muscle and joint pain, muscle spasms (Chopra,1986) sore throat, ulcerative colitis (Kaul, 1997). In folklore medicines, the plant is used for several inflammatory disorders such as arthritis, asthma, conjunctivitis, encephalitis, pancreatitis, peritonitis, acute infections and neuro inflammatory disorders (Shanafelt et al., 2002). Al A l acuminata serve as one of the most important source of medicinally important tropane alkaloids, including atropine, scopolamine and hyoscyamine (Nisar et al., 2013). The drugs atropine and hyoscyamine extracted from the plant act as stimulants to the sympathetic nervous system and are employed as antidotes to opium (Phillipson and Handa, 1975). A. acuminata contains highly oxygenated oleanane triterpenes such as $2 \alpha, 3 \alpha, 24-$ trihydroxyolean-12-ene-28, 30-dioic acid and $2 \alpha, 3 \alpha$, 24, 28-tetrahydroxyolean-12-ene (Mehmood et al., 2002). Monoterpene, sesquiterpene, phenylpropanoid, flavonoid and quinine are present as main constituents (Jayakanthi et al., 2011).

\section{Materials and Methods}

Petiole from in vitro raised plants was used for the experimental purpose. In vitro petiole explants were cultured on MS basal medium, MS medium supplemented with different concentrations and combinations of plant growth regulators both individually and in combinations. Auxins like 2, 4- D; IAA; NAA; IBA and cytokinins like BAP and Kn were used in concentration range of $0.1-5 \mathrm{mg} / \mathrm{L}$. The 
$\mathrm{pH}$ of the medium was adjusted to 5.8 prior to gelling with agar was dispensed in culture tubes and flasks and sterilized by autoclaving at $121^{\circ} \mathrm{C}$ temperature and $15 \mathrm{lbs}$ pressure for 15 minutes. The cultures were incubated under controlled conditions in the culture room under the regime of $16 \mathrm{~h}$ light period (500-3000 lux) and $8 \mathrm{~h}$ dark period and temperature of $22 \pm 4 \mathrm{C}^{\circ}$.

\section{Results and Discussion}

The present study focuses on micropropagation of Atropa acuminata from in vitro petiole explant. Callus was induced when in vitro petiole explants were inoculated on MS medium supplemented with BAP
$(3 \mathrm{mg} / \mathrm{l})$, BAP $(3 \mathrm{mg} / \mathrm{l})+\mathrm{IAA}(2 \mathrm{mg} / \mathrm{l}), \mathrm{BAP}(5 \mathrm{mg} / \mathrm{l})+$ IAA $(1 \mathrm{mg} / \mathrm{l})$ and BAP $(5 \mathrm{mg} / \mathrm{l})+\mathrm{IAA}(2 \mathrm{mg} / \mathrm{l})($ Fig. $)$ in $80 \%, 50 \%, 30 \%$ and $40 \%$ cultures within $20,29,32$ and 30 days. Best response was observed on MS medium enriched with BAP at a concentration of $(3 \mathrm{mg} / \mathrm{L})$ in terms of percent culture response and minimum number of days taken (Fig.1 and table 1). Similar results were also obtained by Amin et al., (2017) from in vitro petiole explant on MS medium augmented with BAP (1mg/l) and $\mathrm{Kn}(1 \mathrm{mg} / \mathrm{l})$.
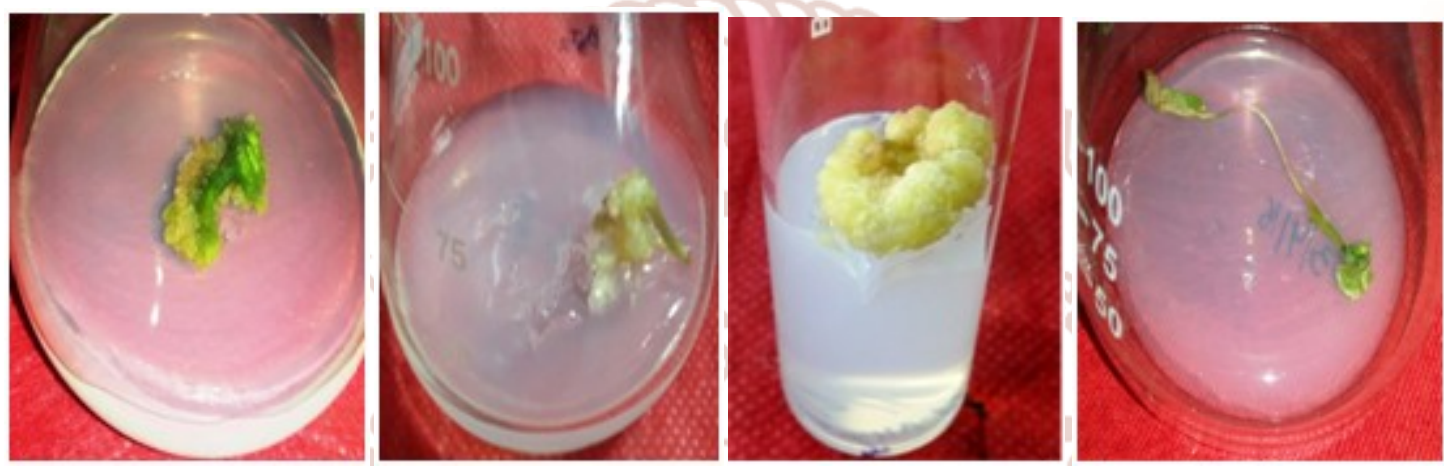

Fig.1: callus production from in vitro petiole explant on MS medium containing

a) $\operatorname{BAP}(3 \mathrm{mg} / 1)$

b) $\operatorname{BAP}(3 \mathrm{mg} / \mathrm{l})+\operatorname{IAA}(2 \mathrm{mg} / \mathrm{l})$

c) $\operatorname{BAP}(5 \mathrm{mg} / 1)+\mathrm{IAA}(1 \mathrm{mg} / \mathrm{l})$

d) $\operatorname{BAP}(5 \mathrm{mg} / \mathrm{l})+\mathrm{IAA}(2 \mathrm{mg} / \mathrm{l})$

Table 1: Effect of plant growth regulators on callus induction from in vitro petiole explant

\begin{tabular}{|c|c|c|c|}
\hline Treatments & $\begin{array}{l}\text { Number of } \\
\text { days taken } \\
\text { for callus } \\
\text { production }\end{array}$ & $\begin{array}{l}\text { Texture } \\
\text { and color } \\
\text { of callus }\end{array}$ & $\begin{array}{l}\% \\
\text { culture } \\
\text { response }\end{array}$ \\
\hline MS basal & - & - & 7 \\
\hline $\begin{array}{l}\mathrm{MS}+\mathrm{BAP} \\
(3 \mathrm{mg} / \mathrm{l})\end{array}$ & 20 & $\begin{array}{l}\text { Hard and } \\
\text { light } \\
\text { brown }\end{array}$ & \\
\hline $\begin{array}{l}\mathrm{MS}+\mathrm{BAP} \\
(3 \mathrm{mg} / \mathrm{l})+ \\
\mathrm{IAA}(2 \mathrm{mg} / \mathrm{l})\end{array}$ & 29 & $\begin{array}{l}\text { Hard and } \\
\text { creamish }\end{array}$ & 50 \\
\hline $\begin{array}{l}\mathrm{MS}+\mathrm{BAP} \\
(5 \mathrm{mg} / \mathrm{l})+ \\
\mathrm{IAA}(1 \mathrm{mg} / \mathrm{l})\end{array}$ & 32 & $\begin{array}{l}\text { Compact } \\
\text { and } \\
\text { creamish }\end{array}$ & 30 \\
\hline $\begin{array}{l}\mathrm{MS}+\mathrm{BAP} \\
(5 \mathrm{mg} / \mathrm{l})+ \\
\mathrm{IAA}(2 \mathrm{mg} / \mathrm{l})\end{array}$ & 30 & $\begin{array}{l}\text { Fragile } \\
\text { and light } \\
\text { green }\end{array}$ & 40 \\
\hline
\end{tabular}

10 replicates per treatment

In vitro petiole callus when subcultured on MS medium supplemented with BAP $(3 \mathrm{mg} / \mathrm{l})$, BAP $(4 \mathrm{mg} / \mathrm{l})$, BAP $(5 \mathrm{mg} / \mathrm{l})$, BAP $(3 \mathrm{mg} / \mathrm{l})+\mathrm{IAA}(1 \mathrm{mg} / \mathrm{l})$, BAP $(3 m g / 1)+\operatorname{IAA}(2 m g / 1)$, BAP $(3 m g / 1)+$ IAA $(3 \mathrm{mg} / 1), \mathrm{BAP}(3 \mathrm{mg} / \mathrm{l})+\mathrm{IAA}(4 \mathrm{mg} / \mathrm{l}), \mathrm{BAP}(3 \mathrm{mg} / \mathrm{l})+$ $\mathrm{Kn}(1 \mathrm{mg} / \mathrm{l}), \mathrm{BAP}(3 \mathrm{mg} / \mathrm{l})+\mathrm{Kn}(2 \mathrm{mg} / \mathrm{l}), \mathrm{BAP}(3 \mathrm{mg} / \mathrm{l})$ $+\mathrm{Kn}(3 \mathrm{mg} / \mathrm{l})$, BAP $(3 \mathrm{mg} / \mathrm{l})+\mathrm{Kn}(4 \mathrm{mg} / \mathrm{l})$ and BAP $(3 \mathrm{mg} / \mathrm{l})+\mathrm{Kn}(5 \mathrm{mg} / \mathrm{l})$ (Fig.) regenerate shoots with $8.6 \pm 4.22 \mathrm{~cm}, 5.6 \pm 2.69 \mathrm{~cm}, 4.8 \pm 2.35 \mathrm{~cm}, 3.0 \pm 0.70 \mathrm{~cm}$, $7.2 \pm 3.45 \mathrm{~cm}$ and $5.0 \pm 2.07 \mathrm{~cm}$ mean number of shoots and $\quad 2.3 \pm 0.20 \mathrm{~cm}, \quad 3.9 \pm 0.20 \mathrm{~cm}, \quad 2.7 \pm 0.15 \mathrm{~cm}$, $4.6 \pm 0.29 \mathrm{~cm}, \quad 1.4 \pm 0.16 \mathrm{~cm}$ and $1.5 \pm 0.18 \mathrm{~cm}$ mean length of shoots in $100 \%, 80 \%, 60 \%, 20 \%, 70 \%$, $40 \%, 30 \%, 20 \%, 50 \%, 70 \%, 40 \%$ and $30 \%$ cultures within 13, 18, 24, 29, 16, 20, 42, 35, 26, 17, 32 and 25 days respectively. Maximum number of shoots was obtained with a mean shoot number of $8.6 \pm 4.22 \mathrm{~cm}$ and mean shoot length of $2.3 \pm 0.20 \mathrm{~cm}$ when BAP at a concentration of $(3 \mathrm{mg} / \mathrm{L})$ was added to the medium (Fig. 2 and Table. 2). This is the first report of shoot regeneration from in vitro petiole explant. 

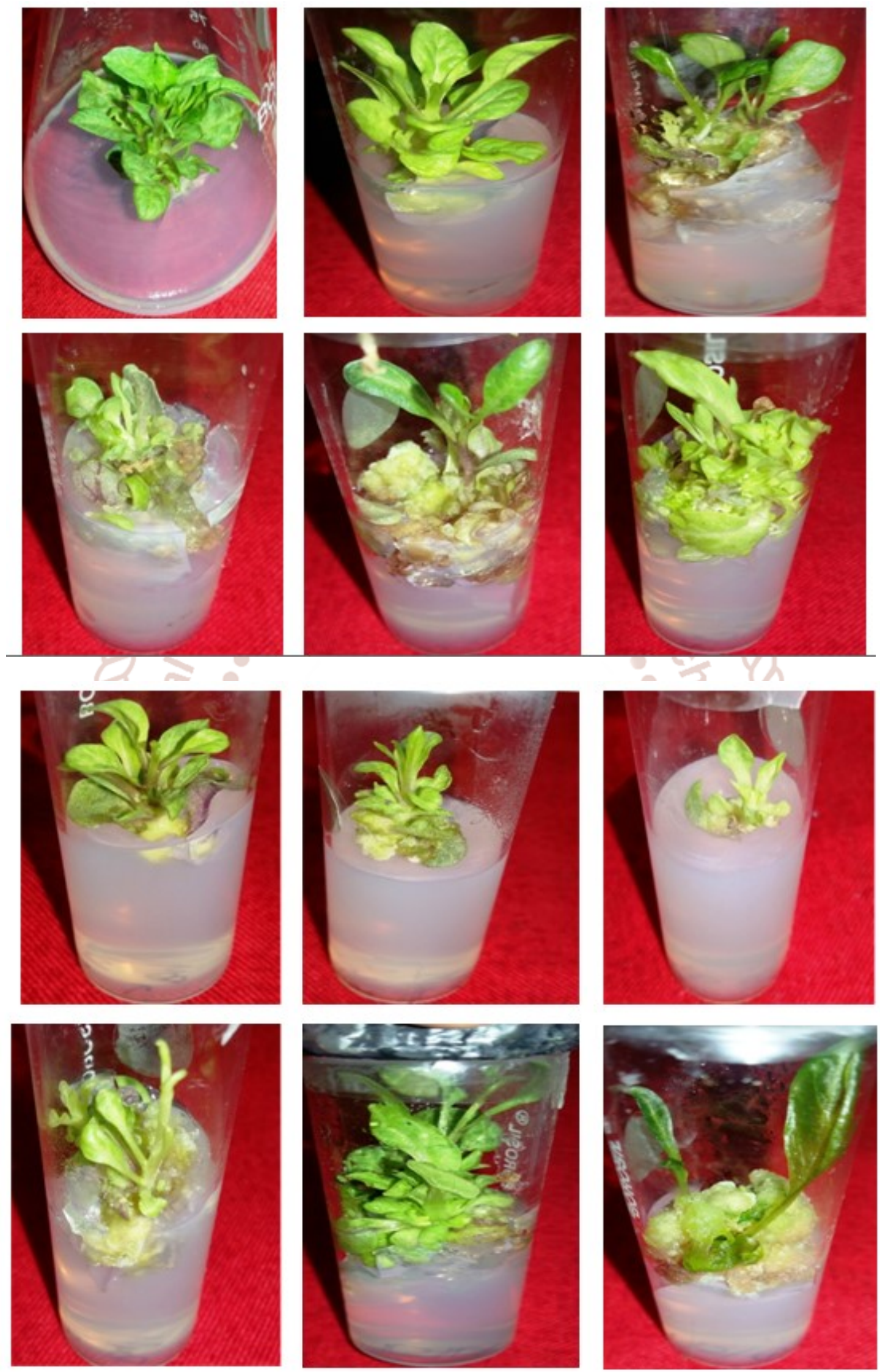

Fig.2: Shoot regeneration from in vitro petiole explant on MS medium containing

a) BAP (3mg/l) b) BAP (4mg/l) c) BAP (5mg/l) d) BAP (3mg/l) + IAA (1mg/l) e) BAP (3mg/l) + IAA $(2 \mathrm{mg} / \mathrm{l}) \mathrm{f}) \mathrm{BAP}(3 \mathrm{mg} / \mathrm{l})+\mathrm{IAA}(3 \mathrm{mg} / \mathrm{l}) \mathrm{g}) \mathrm{BAP}(3 \mathrm{mg} / \mathrm{l})+\mathrm{IAA}(4 \mathrm{mg} / \mathrm{l}) \mathrm{h}) \mathrm{BAP}(3 \mathrm{mg} / \mathrm{l})+\mathrm{Kn}(1 \mathrm{mg} / \mathrm{l}) \mathrm{i})$ 
International Journal of Trend in Scientific Research and Development (IJTSRD) ISSN: 2456-6470

$\operatorname{BAP}(3 m g / 1)+K n(2 m g / l) j) \operatorname{BAP}(3 m g / l)+K n(3 m g / l) k)$ BAP $(3 m g / l)+K n(4 m g / l) 1)$ BAP $(3 m g / l)+$ $\mathrm{Kn}(5 \mathrm{mg} / \mathrm{l})$

Table 2: Effect of plant growth regulators on shoot regeneration from in vitro petiole derived callus

\begin{tabular}{|c|c|c|c|c|}
\hline Treatments & $\begin{array}{c}\text { Mean number of } \\
\text { days taken for } \\
\text { shoot regeneration }\end{array}$ & $\begin{array}{c}\text { Mean number } \\
\text { of shoots } \\
\text { (cm) } \pm \text { SE }\end{array}$ & $\begin{array}{l}\text { Mean length } \\
\text { of shoots } \\
(\mathrm{cm}) \pm \mathrm{SE}\end{array}$ & $\begin{array}{l}\% \text { culture } \\
\text { response }\end{array}$ \\
\hline $\mathrm{MS}+\mathrm{BAP}(3 \mathrm{mg} / \mathrm{l})$ & 13 & $8.6 \pm 4.22$ & $2.3 \pm 0.20$ & 100 \\
\hline $\mathrm{MS}+\mathrm{BAP}(4 \mathrm{mg} / \mathrm{l})$ & 18 & $5.6 \pm 2.69$ & $3.9 \pm 0.20$ & 80 \\
\hline $\mathrm{MS}+\mathrm{BAP}(5 \mathrm{mg} / \mathrm{l})$ & 24 & $4.8 \pm 2.35$ & $2.7 \pm 0.15$ & 60 \\
\hline $\begin{array}{c}\mathrm{MS}+\mathrm{BAP}(3 \mathrm{mg} / \mathrm{l})+\mathrm{IAA} \\
(1 \mathrm{mg} / \mathrm{l})\end{array}$ & 29 & $3.0 \pm 0.70$ & $4.6 \pm 0.29$ & 20 \\
\hline $\begin{array}{c}\mathrm{MS}+\mathrm{BAP}(3 \mathrm{mg} / \mathrm{l})+\mathrm{IAA} \\
(2 \mathrm{mg} / \mathrm{l})\end{array}$ & 16 & $5.2 \pm 3.45$ & $1.4 \pm 0.16$ & 70 \\
\hline $\begin{array}{c}\mathrm{MS}+\mathrm{BAP}(3 \mathrm{mg} / \mathrm{l})+\mathrm{IAA} \\
(3 \mathrm{mg} / \mathrm{l})\end{array}$ & & 010 & $1.5 \pm 0.18$ & 40 \\
\hline $\begin{array}{c}\mathrm{MS}+\mathrm{BAP}(3 \mathrm{mg} / \mathrm{l})+\mathrm{IAA} \\
(4 \mathrm{mg} / \mathrm{l})\end{array}$ & & $3.4 \pm 1.02$ & $1.1 \pm 0.14$ & 30 \\
\hline $\begin{array}{c}\mathrm{MS}+\mathrm{BAP}(3 \mathrm{mg} / \mathrm{l})+\mathrm{Kn} \\
(1 \mathrm{mg} / \mathrm{l})\end{array}$ & 35 & $1.8 \pm 0.58$ & $0.7 \pm 0.22$ & 20 \\
\hline $\begin{array}{c}\mathrm{MS}+\mathrm{BAP}(3 \mathrm{mg} / \mathrm{l})+\mathrm{Kn} \\
(2 \mathrm{mg} / \mathrm{l})\end{array}$ & 8 & $2.2 \pm 0.73$ & $0.9 \pm 0.26$ & 50 \\
\hline $\begin{array}{c}\mathrm{MS}+\mathrm{BAP}(3 \mathrm{mg} / \mathrm{l})+\mathrm{Kn} \\
(3 \mathrm{mg} / \mathrm{l})\end{array}$ & nte 17 & $7.8 \pm 3.69$ & $4.9 \pm 0.19$ & 80 \\
\hline $\begin{array}{c}\mathrm{MS}+\mathrm{BAP}(3 \mathrm{mg} / \mathrm{l})+\mathrm{Kn} \\
(4 \mathrm{mg} / \mathrm{l})\end{array}$ & 32 no If & $S \mathrm{C} 1.8 \pm 0.58 \mathrm{C}$ & $3.3 \pm 0.94$ & 40 \\
\hline $\begin{array}{c}\mathrm{MS}+\mathrm{BAP}(3 \mathrm{mg} / \mathrm{l})+\mathrm{Kn} \\
(5 \mathrm{mg} / \mathrm{l})\end{array}$ & $\begin{array}{l}25 \\
\text { Devere }\end{array}$ & $3.4 \pm 1.02$ & $0.8 \pm 0.09$ & 30 \\
\hline
\end{tabular}

\section{0 replicates per treatment}

\section{Rooting of regenerated shoots from in vitro petiole explant}

Roots were regenerated from the shoots of in vitro petiole explant inoculated on full strength MS medium. Roots were also regenerated on MS medium supplemented with IBA (0.2mg/1) and IBA (0.5mg/l) (Fig. 25) with $2.2 \pm 0.37 \mathrm{~cm}, 3.6 \pm 1.20 \mathrm{~cm}$ and $6.0 \pm 2.50 \mathrm{~cm}$ mean number of roots and $1.3 \pm 0.11 \mathrm{~cm}, 2.6 \pm 0.20 \mathrm{~cm}$ and $1.3 \pm 0.11 \mathrm{~cm}$ mean length of roots in $40 \%, 30 \%$ and $60 \%$ cultures within 38,48 and 32 days of inoculation respectively (Fig. 3 and Table 3 ).
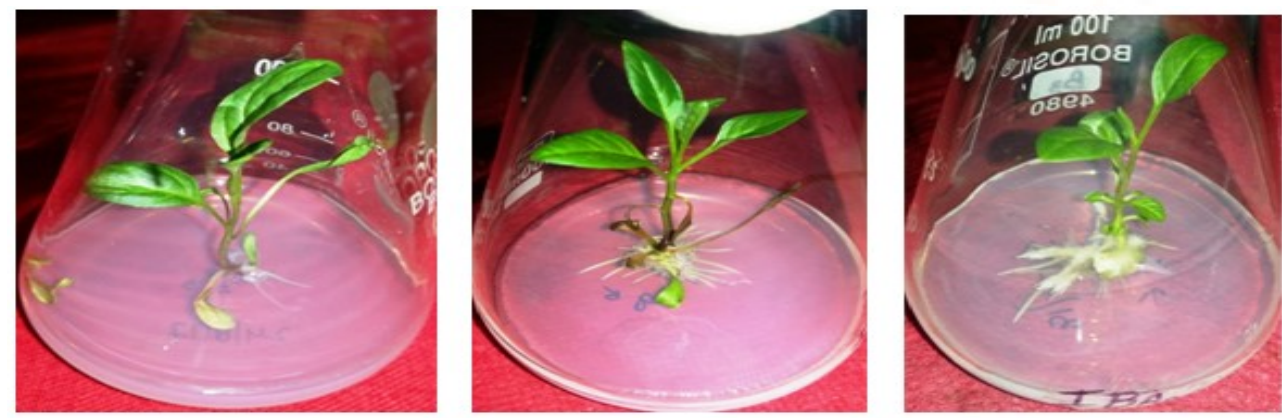

Fig.3: Rooting of regenerated shoots from in vitro petiole explant
a) MS basal
b) IBA $(0.2 \mathrm{mg} / \mathrm{l})$
c) IBA $(0.5 \mathrm{mg} / \mathrm{l})$

Table 26: Effect of plant growth regulators on rooting of regenerated shoots from in vitro petiole explant 
International Journal of Trend in Scientific Research and Development (IJTSRD) ISSN: 2456-6470

\begin{tabular}{|c|c|c|c|c|}
\hline Treatments & $\begin{array}{c}\text { Number of days } \\
\text { taken for root } \\
\text { regeneration }\end{array}$ & $\begin{array}{c}\text { Mean number } \\
\text { of roots } \\
(\mathbf{c m}) \pm \text { SE }\end{array}$ & $\begin{array}{c}\text { Mean length } \\
\text { of roots } \\
(\mathbf{c m}) \pm \text { SE }\end{array}$ & $\begin{array}{c}\% \text { culture } \\
\text { response }\end{array}$ \\
\hline MS basal & 38 & $2.2 \pm 0.37$ & $1.3 \pm 0.11$ & 40 \\
\hline MS+IBA $(0.2 \mathrm{mg} / \mathrm{l})$ & 48 & $3.6 \pm 1.20$ & $2.6 \pm 0.20$ & 30 \\
\hline MS+IBA $(0.5 \mathrm{mg} / \mathrm{l})$ & 32 & $6.0 \pm 2.50$ & $1.3 \pm 0.11$ & 60 \\
\hline
\end{tabular}

\section{0 replicates per treatment}

\section{REFERENCES}

1. Nasir, Y. J. (1972) Atropa acuminata Royle ex miers in Hook Flora of Pakistan. 1:138.

2. Rhodes, J. B., Abrams, J. H., Manning, R. T. (1978) Controlled clinical trial of sedativeanticholinergic drugs in patients with the irritable bowel syndrome. Journal of Clinical Pharmacology, 18: 340-345.

3. Grieve, (1984) A Modern Herbal. Pengium ISBN 0-14-046-440-9.

4. Chiej, R. (1984) The Macdonald encyclopedia of medicinal plants. Macdonald \& Co (Publishers) Ltd.

5. Chopra, R. N., Nayar. S. L., Chopra. I. C. (1986) Glossary of Indian medicinal plants (Including the Supplement) Council of Scientific and Industrial Research, New Delhi.

6. Kaul, M. K. (1997) Medicinal plants of Kashmir and Ladakh. Indus Publications, New Delhi, India pp. 173.
7. Shanafelt, T. D., Barton, D. L., Adjei, A. A., Loprinzi, C. L. (2002) Path physiology and treatment of hot flashes In: Mayo Clinic proceedings. Mayo Clinic, 77: 1207-1218.

8. Nisar, A., Malik, A. H., Zargar, M. A. (2013) Atropa acuminata blunts production of proinflammatory mediator's eicosanoids, leukotrienes, cytokines in vitro and in-vivo models of acute inflammatory responses. Journal of Ethnopharmacology, 147:584-594.

9. Phillipson, J. D., Handa, S. S. (1975) N-oxides of hyoscyamine and hyoscine in the Solanaceae Photochemistry, 14: 999-1003.

10. Mehmood, M. A., Anis, I., Khan, P. M., Riaz, M., Makhmoor, T., Choudhary, M. I. (2002) Highly oxygenated triterpenes from the roots of Atropa acuminata. Natural Product Letters, 16: 371-376.

11. Jayakanthi, J., Dhanarajan, M. S., Vijay, T. (2011) Found main constituents of Atropa acuminata belongs to monoterpene, sesquiterpene, phenylpropanoid, flavonoid and quinine International Journal of Pharmacy and Pharmaceutical Sciences, 3: 0975-1491. 